\title{
WHAT CAN BE EXPECTED FROM LEAD-FREE PIEZOELECTRIC MATERIALS?
}

\author{
DRAGAN DAMJANOVIC*, NAAMA KLEIN, JIN LI \\ and VIKTOR POROKHONSKYY \\ Ceramics Laboratory, Swiss Federal Institute of Technology \\ EPFL CH-1015 Lausanne, Switzerland \\ *dragan.damjanovic@epfl.ch
}

Received 2 December 2009; Revised 2 January 2010

\begin{abstract}
The reasons for the lower piezoelectric properties in the most studied lead-free piezoelectrics, modified $(\mathrm{K}, \mathrm{Na}) \mathrm{NbO}_{3}$ and $\left(\mathrm{Bi}_{0.5} \mathrm{Na}_{0.5}\right) \mathrm{TiO}_{3}$, are discussed. Contributions from domain wall motion and properties at the morphotropic phase boundary are considered and are compared to those in PZT. Lead-free, non-piezoelectric solutions to electromechanical coupling are discussed.
\end{abstract}

Keywords: Lead-free; piezoelectrics; ferroelectrics; sodium-bismuth titanate; potassium niobate.

\section{Introduction}

With few exceptions (e.g. bulk acoustic wave resonators in $\mathrm{GHz}$ range, surface acoustic wave devices, frequency control, high precision pressure sensors), the materials that are presently best suited for the vast majority of piezoelectric devices are based on solid solutions of lead-based oxides: lead zirconate titanate or $\mathrm{Pb}\left(\mathrm{Zr}_{1-x} \mathrm{Ti}_{x}\right) \mathrm{O}_{3}$ (PZT) and relaxor-ferroelectrics e.g. $(1-x) \mathrm{Pb}\left(\mathrm{Mg}_{1 / 3} \mathrm{Nb}_{2 / 3}\right) \mathrm{O}_{3}$ $x \mathrm{PbTiO}_{3}$ (PMN-PT). ${ }^{1}$ It is extraordinary that one family PZT, with some chemical modifications, is by far the most widely used piezoelectric material. Considering performance, cost, ease of preparation and precursors availability PZT is the almost perfect all-around piezoelectric material. ${ }^{2}$

However, because of lead toxicity, ${ }^{3,4}$ alternatives to $\mathrm{Pb}$ based materials are being intensively sought. Interest in leadfree piezoelectrics has been expressed in the 1950s, when PZT was discovered. But it is only recently, with the increased environmental awareness and legislatively-backed concerns, ${ }^{5}$ that intensive world-wide activities on development of lead-free piezoelectrics have been initiated. ${ }^{6-8}$ Two families of leadfree materials have presently emerged as the most promising candidates to replace PZT: modified potassium sodium niobate $(\mathrm{K}, \mathrm{Na}) \mathrm{NbO}_{3}(\mathrm{KNN})$ and bismuth sodium titanate $\left(\mathrm{Bi}_{0.5} \mathrm{Na}_{0.5}\right) \mathrm{TiO}_{3}$ (BNT) ${ }^{9}, 10$ Properties of these materials are interesting but overall, they do not match those of PZT. Despite some success in demonstrating performance acceptable for

*Corresponding Author. diverse devices, such as ultrasonic motors, ${ }^{11}$ high frequency transducers ${ }^{12}$ and buzzers, ${ }^{13}$ one is still far from the ideal goal of having a lead-free family with wide spectrum of high properties as exhibited by PZT. ${ }^{14}$

Without denying toxicity of lead compounds, questions have been raised about the need to replace PZT, considering uncontested benefits of PZT-based piezoelectric devices (e.g. in medical ultrasound and therapy). Arguments have been given indicating low probability that lead may enter environment at the end-of-life of many piezoelectric devices. ${ }^{7}$

Apart from these environmental/societal and technological/commercial issues that drive much of the present activities, high-performance lead-free piezoelectrics are of a great scientific interest in the fields of materials physics and chemistry today. In this article, these scientific issues are discussed, in particular reasons why PZT is such exceptional piezoelectric material. Indications are given why presently considered lead-free materials are inferior to PZT and how they can be improved. Lead-free alternatives to the piezoelectric effect, including electrostriction and a more radical solution, flexoelectric effect, are briefly discussed.

\section{Why is PZT a Good Piezoelectric Material?}

Under usual operating conditions (ambient temperature, atmospheric environmental pressure, subcoercive driving fields and pressures), the best performing piezoelectric materials are those that contain lead atom (e.g. PZT, PMN-PT). The first 
principle calculations suggest that outstanding ferroelectric properties of $\mathrm{Pb}$-based materials are related to the particular electronic structure of $\mathrm{Pb}$ (lone $6 \mathrm{~s}^{2}$ pair) and $\mathrm{Pb}-\mathrm{O}$ bond hybridization. ${ }^{15-18}$ However, while presence of $\mathrm{Pb}$ might be a reason, it is clearly not a sufficient "ingredient" for high piezoelectricity. The end members of PZT solid solution are either non-piezoelectric (lead-zirconate, $\mathrm{PbZrO}_{3}$ ) or exhibit moderate piezoelectricity (lead titanate, $\mathrm{PbTiO}_{3}$ ). ${ }^{2}$ Two main reasons why PZT solid solution possesses exceptional piezoelectric properties are: (i) presence of the morphotropic phase boundary (MPB) near $\mathrm{Zr:Ti}=52: 48$, and (ii) high contribution to the electromechanical properties from the motion of ferroelectric domain walls. ${ }^{2}$

Presently, the first reason is taken by the majority of the researchers as the most important and much efforts in the search for lead-free systems with high properties have been focused on solid solutions exhibiting an MPB. ${ }^{9,19}$ In the MPB region of PZT, the ferroelectric polarization changes crystallographic direction from the $\langle 111\rangle$ (rhombohedral, Zr-rich compositions) to $\langle 001\rangle$ pseudocubic axis (tetragonal, Ti-rich compositions) $)^{2}$ via an intermediary monoclinic phase. ${ }^{20}$ This compositionally induced phase transition is accompanied by a large susceptibility to polarization rotation (i.e. transverse permittivity, $\varepsilon_{11}$, and shear piezoelectric coefficients, e.g. $d_{15}$, are large) and to a lesser degree to polarization extension (the permittivity along polar axis, $\varepsilon_{33}$ and the longitudinal piezoelectric coefficient $d_{33}$ are large). ${ }^{21,22}$ It is possible to trace origin of the properties enhancement in the MPB region to the anisotropic flattening of the PZT's free energy. ${ }^{23,24}$ The high properties are sometimes associated with the monoclinic phase but such interpretations are supported with little evidence. ${ }^{25}$

Surprisingly, even though domain walls contribution may account for as much as $70 \%$ of the dielectric and piezoelectric properties in PZT, this aspect has not been widely studied in lead-free candidates. The question that can be posed is whether $\mathrm{Pb}$ cation is needed for special properties at MPB and for large contribution of domain walls motion to the electromechanical coupling.

We first discuss the need for $\mathrm{Pb}$ cation in MPB systems. Recent $a b$ initio and experimental structural studies claim that the origin of the MPB behavior in PT-based ferroelectrics is indeed found in special properties of lead-titanate. These studies show that $\mathrm{PbTiO}_{3}$ undergoes phase transitions into monoclinic, rhombohedral and cubic phases under increasing hydrostatic pressure applied at $0 \mathrm{~K}^{26,27}$ It is suggested that in modified materials (i.e. $\mathrm{PZT}, \mathrm{Pb}\left(\mathrm{Mg}_{1 / 3} \mathrm{Nb}_{2 / 3}\right) \mathrm{O}_{3}-\mathrm{PT}$, $\left.\mathrm{Pb}\left(\mathrm{Zn}_{1 / 3} \mathrm{Nb}_{2 / 3}\right) \mathrm{O}_{3}-\mathrm{PT}\right)$ the role of the external hydrostatic pressure is taken by modifying cations ( $\mathrm{Zr}, \mathrm{Mg}, \mathrm{Nb}$ and $\mathrm{Zn}$ ). It is proposed that these atoms apply an internal "chemical pressure" within the crystal lattice of PT and shift MPB to ambient temperature. The authors propose substitution of $\mathrm{Pb}$ with smaller Sn cation to induce internal compressive pressure, ${ }^{27}$ so it is not clear what would be the role of cations with a larger radius than $\mathrm{Ti}$ ( $\mathrm{Zn}, \mathrm{Nb}, \mathrm{Zr}$ ). More importantly, the hydrostatic pressure is accompanied by the flattening of the free energy profile in the phase transition region, leading to the enhanced properties. If, as claimed by the authors, this mechanism explains origin of the MPB in a general case, then one can consider the following possibilities: (i) the MPBs are confined to PT based materials only or (ii) lead-free systems with an MPB might have to include an end member whose structure can be modified by external pressure, as in $\mathrm{PbTiO}_{3}$.

As we shall see in the next section, at least two lead-free families (modified KNN and BNT) exhibit MPBs. There is little regularity in the behavior of the end-members of various MPB systems that would point out selection rules on which systems may show an MPB. Thus, PT is strongly tetragonal and remains as such from the Curie temperature to $0 \mathrm{~K}$ under atmospheric pressure. $\mathrm{KNN}, \mathrm{KNbO}_{3}(\mathrm{KN})$ and barium titanate or $\mathrm{BaTiO}_{3}$ (BT; used to modify BNT), transform into a series of ferroelectric phases on cooling from the paraelectric phase. Application of the compressive pressure at $0 \mathrm{~K}$ has been predicted to reinforce the ferroelectricity in both BT and PT. ${ }^{28}$ Moreover, there are indications that the free-energy flattening as the origin of the properties enhancement in the MPB region is common to a wide class of materials, including nonferroelectric lead-free systems, such as AlN-ScN solid solution. ${ }^{29}$ Thus, lead cation does not seem to be essential for MPB.

Concerning contributions of domain walls motion to the electromechanical properties, it is not immediately clear why these should in general be higher in $\mathrm{Pb}$-based than lead-free materials. ${ }^{30}$ One possibility might be found in the concept of softening which is well-known in PZT but few examples exist for lead free materials. ${ }^{9,10}$ In perovskite ferroelectrics such as PZT, donor doping on cation sites leads to materials with higher permittivity, piezoelectric coefficients and elastic compliances. The mechanisms of this "softening" are not well understood. One qualitative model relates softening to the presence of $\mathrm{Pb}$ vacancies $\left(\mathrm{V}_{\mathrm{Pb}}\right)$ using the following speculative argument: ${ }^{31}$ in donor doped PZT, the charge compensating defects are $\mathrm{V}_{\mathrm{Pb}}$. It is proposed that presence of $\mathrm{V}_{\mathrm{Pb}}$ releases the stress build-up associated with motion of non- $180^{\circ}$ domain walls and results in their easier displacement. This may not happen in lead-free materials, such as BT, because Ba is not as volatile as $\mathrm{Pb}$, and $\mathrm{BT}$ compensates donor dopants by electrons rather than by cation vacancies. In materials containing $\mathrm{Bi}$ and alkaline metals, the A-site vacancy concentration may be substantial. However, in these materials other properties may deteriorate with a high concentration of cation vacancies so that the softening effect is lost. Thus, one might speculate that the properties of lead atom may indeed be the key to softening of PZT. 
Finally, it is interesting to consider combined effects of MPB and domain walls in this region where two phases meet and free energy profile of the system becomes flatter. It can be inferred from the phenomenological theory that domain walls are not essential for the enhanced properties in the MPB region because monodomain single crystals of PZT also exhibit enhanced properties at MPB. ${ }^{21,32}$ Nevertheless, the large piezoelectric response of PZT lattice at MPB is further enhanced by the domain wall contributions, which are also particularly strong in this region. ${ }^{2,30}$ The reason for the high domain walls contributions at MPB is likely related to the general flattening of the free energy profile in the transition region. In addition, it was shown recently that in the vicinity of MPB, the domain size in PZT becomes very fine, with domain widths (distance between the domain walls) on the order of tens of nanometers. ${ }^{33,34}$ The nanodomains probably form to accommodate stress build-up caused by coexistence of tetragonal and rhombohedral phases. A suggestion has been made that the fine domain walls structure is associated with the enhanced properties. However, there is not much direct evidence that a fine domains structure may be the dominant factor responsible for the enhanced properties. ${ }^{35}$

Taking PZT as a reference, we now discuss properties of some lead-free materials.

\section{Lead-Free Piezoelectrics and Alternatives to PZT}

There are many lead-free piezoelectric materials, some of which (quartz, $\mathrm{LiTaO}_{3}, \mathrm{PVDF}$, some bismuth titanate based materials) have firmly established place in special applications. Due to their low piezoelectric and coupling coefficients and considering the vast spectrum of applications of PZT, these materials cannot be considered as alternatives for the entire PZT family. Some other lead free materials that are presently being considered are, like those listed above, complementary to PZT and can be of interest only for specific applications. Examples include oxyborates for operation at high temperatures ${ }^{36}$ and AlN for high frequencies ${ }^{37}$ In recent years, efforts were being made to develop multiferroic leadfree materials, such as $\mathrm{BiFeO}_{3}{ }^{38}$ and solid solutions of other Bi-based perovskites (e.g. $\mathrm{BiScO}_{3}, \mathrm{BiAlO}_{3}$ ). ${ }^{39,40} \mathrm{Bi}$-based perovskites are of special interest because of similarities between $\mathrm{Bi}$ and $\mathrm{Pb}$ atoms. However, neither empirical studies nor ab initio calculations have led to a Bi-based, lead-free system with properties comparable to those of PZT. ${ }^{18,40}$ Other materials, such as Seignette's (Rochelle) salt $\left(\mathrm{KNaCH}_{4} \mathrm{O}_{6}\right.$. $4 \mathrm{H}_{2} \mathrm{O}$ ) and $\mathrm{KH}_{2} \mathrm{PO}_{4}(\mathrm{KDP})$ exhibit as high or even much higher piezoelectric properties than ZT but are impractical due to chemical instability or low temperatures required for operation. ${ }^{25,41}$
Next, we shall discuss three families of lead-free materials which are at present considered as the best candidates to replace PZT in a somewhat broader range of applications: BT and modified BNT and KNN. All three base materials were discovered in the 1940s-1960s and compositions used today are usually heavily modified chemically. Non-modified barium titanate is being revisited, and rather spectacular piezoelectric properties have been reported in textured ceramics ${ }^{42}$ and ceramics derived from nanopowders. ${ }^{43}$

In the most widely studied lead-free system, $(\mathrm{K}, \mathrm{Na}, \mathrm{Li})$ $(\mathrm{Nb}, \mathrm{Ta}) \mathrm{O}_{3}$ (modified KNN) developed by the Toyota Central Research Laboratory, ${ }^{6}$ the large properties at room temperature appear to be caused by the proximity of the temperature induced phase transition between tetragonal and orthorhombic phases to the ambient temperature. ${ }^{10}$ The temperature of this polymorphic phase transition depends on composition. The boundary between the two phases is thus not vertical in the composition-temperature phase diagram and has mixed MPB and polymorphic character. As a consequence, there is a significant thermal instability of the properties when a sample is cooled or heated from room temperature. ${ }^{44}$ Modifications of KNN having better temperature stability have been proposed. ${ }^{45}$ However, the competing contributions of the MPB and the thermally induced phase instabilities to the properties enhancement have not been elucidated. This is a serious drawback for further development of this material because tuning of the properties for specific applications requires an in-depth understanding of the structure-property relations. In the case of $\mathrm{KNN}$, there is no consensus on whether the crystal structure of the base composition $(\mathrm{K}, \mathrm{Na}) \mathrm{NbO}_{3}$ is orthorhombic or monoclinic ${ }^{46}$ (see also contribution by Kosec et al. in this issue).

The longitudinal piezoelectric coefficient in KNN based materials has been reported to reach as much as $400 \mathrm{pC} / \mathrm{N}$ in textured $(\mathrm{K}, \mathrm{Na}, \mathrm{Li})(\mathrm{Nb}, \mathrm{Ta}, \mathrm{Sb}) \mathrm{O}_{3}$ ceramics, ${ }^{6}$ but more commonly reported values range from 100 to $250-300 \mathrm{pC} / \mathrm{N}^{47-49}$ These values should be compared to $200-600 \mathrm{pC} / \mathrm{N}$ in commercial PZT.

A vertical MPB has been discovered in $\left(\mathrm{K}_{0.5} \mathrm{Na}_{0.5}\right)_{1-x}$ $\left.\mathrm{Li}_{x}\right) \mathrm{NbO}_{3} \cdot{ }^{50}$ It appears approximately at $x=0.05$ and below $-100^{\circ} \mathrm{C}$, at temperatures which are of little practical interest. Regardless of this, it would be interesting from a fundamental point of view to know if this MPB leads to enhanced electro-mechanical properties. Unlike MPB in PZT, it separates monoclinic and a pseudo-rhombohedral phase and it is not immediately clear if such a boundary would be effective in enhancing the electromechanical properties. It is worth noting that in non-modified $(\mathrm{K}, \mathrm{Na}) \mathrm{NbO}_{3}$, the MPB at $\mathrm{Na}: \mathrm{K}$ ratio of 50:50, separates two pseudo-orthorhombic phases and it is known that the properties enhancement is less pronounced than in PZT (for example, there is no maximum in the permittivity at this MPB) ${ }^{51}$ All these examples suggest that it 
would be worthwhile to investigate how the crystal structure of the phases on each side of the MPB influences the flattening of a free energy profile. It is important that some studies (see contribution by Kosec et al. in this issue and Ref. 46) index pseudo-orthorhombic phases in KNN as monoclinic. If this is indeed so, then the absence of exceptional electromechanical response throughout this solid solution indicates that monoclinic structure by itself cannot, as sometimes suggested, lead to exceptionally large properties. For a detailed discussion on the relationship between monoclinic phases and large electro mechanical properties see Ref. 25.

In solid solutions based on $\mathrm{Bi}_{0.5} \mathrm{Na}_{0.5} \mathrm{TiO}_{3}$, the situation is even more complex. The $(1-x) \mathrm{Bi}_{0.5} \mathrm{Na}_{0.5} \mathrm{TiO}_{3}-x \mathrm{BaTiO}_{3}$ phase diagram exhibits ferroelectric, relaxor, and several nonpolar phases, ${ }^{52}$ whose nature has not yet been resolved. ${ }^{53}$ The transition between the rhombohedral (BNT-rich) and tetragonal (BT-rich) regions appears at room temperature around $x=5-7 \%$, depending on the source. ${ }^{54}$ The structural analyses with XRD indicate very small tetragonal and rhombohedral distortions up to $9 \%$ BT so that the structure on both sides of the MPB appears as pseudocubic. ${ }^{55,56}$ The dielectric anisotropy and consequently polarization rotation effects are thus expected to be small. An indication that this is indeed the case can be found in the values of the permittivity and piezoelectric coefficients of poled 0.94BNT-0.06BT ceramics: $\varepsilon_{33}^{T}=580$, $\varepsilon_{11}^{T}=730, d_{33}=125 \mathrm{pC} / \mathrm{N}$ and $d_{15}=194 \mathrm{pC} / \mathrm{N} .{ }^{57}$ Note the low dielectric anisotropy ratio, ${ }^{22} \varepsilon_{11}^{T} / \varepsilon_{33}^{T}=1.26$ and the low shear piezoelectric coefficient $d_{15}$. In comparison, the dielectric and piezoelectric anisotropy of poled $\mathrm{PbZr}_{0.54} \mathrm{Ti}_{0.46} \mathrm{O}_{3}$ ceramics is significantly higher: $\varepsilon_{11}^{T}=990, \varepsilon_{33}^{T}=450$, $\varepsilon_{11}^{T} / \varepsilon_{33}^{T}=2.2, d_{33}=152 \mathrm{pC} / \mathrm{N}$ and $d_{15}=440 \mathrm{pC} / \mathrm{N} ;{ }^{58}$ the large shear piezoelectric coefficient in PZT, which is closely related to polarization rotation effects, should be noted. While comparison of the rhombohedral PZT and the tetragonal BNT-BT ceramics may not be justified, the above assessment nevertheless seems to be fair; it has been reported that the piezoelectric $d$ coefficients of BNT-BT ceramics are inferior to those of PZT or even $\mathrm{KNN}$ across the MPB region $\left(d_{33}\right.$ is on the order of $100-200 \mathrm{pC} / \mathrm{N}^{59}$ ). Interestingly, all phase diagrams of BNT-BT published so far give only one, room temperature point on the MPB. ${ }^{52,60}$ The very recent Raman studies by the authors indicate a vertical morphotropic phase boundary at $x=5.5 \%(+/-0.5)$ from above room temperature to nearly $0 \mathrm{~K} .{ }^{50}$ The low depolarization temperature $\left(<150^{\circ} \mathrm{C}\right)$, which indicates onset of a nonpolar phase and loss of piezoelectric response is another problem. The details of this nonpolar structure are not known. Very high piezoelectric coefficients $\left(d_{33}>500 \mathrm{pC} / \mathrm{N}\right)$ have been reported for single crystals of BNT-BT solid solution along a nonpolar direction but not confirmed independently. ${ }^{60,61}$ Large longitudinal piezoelectric coefficient along a nonpolar axis would be incompatible with a low dielectric anisotropy and low transverse permittivity. ${ }^{25}$
It is possible to identify some important differences between KNN and BNT-BT on one and PZT on the other side. These differences may be the possible reasons why piezoelectric properties of these lead-free systems are inferior to those of PZT. In PZT, the MPB separates well-developed rhombohedral and tetragonal phases (with possibly monoclinic bridge between them). ${ }^{20}$ The MPB ends at a tricritical point where rhombohedral and tetragonal ferroelectric phases and paraelectric cubic phase meet. In modified KNN and BNT-BT, the MPBs do not end at the paraelectric cubic phase, and the crystallographic distortion between the two phases which form MPB is much less pronounced than in PZT. It is certainly tempting to assign this difference between PZT and these two lead-free systems to the absence of $\mathrm{Pb}$ in the latter. The following question can then be posed: "whether there are lead-free systems with a PZT-like MPB?"

Liu and Ren claim to have discovered a solid solution that, despite important differences, exhibit some remarkable similarities to PZT. ${ }^{62}$ They reported that $(1-x) \mathrm{Ba}\left(\mathrm{Ti}_{0.8} \mathrm{Zr}_{0.2}\right) \mathrm{O}_{3}$ $x\left(\mathrm{Ba}_{0.7} \mathrm{Ca}_{0.3}\right) \mathrm{TiO}_{3}$ solid solution exhibits an MPB between rhombohedral and tetragonal phases ending up at a tricritical point with a cubic paraelectric phase. The remarkably high piezoelectric coefficient $\left(d_{33}>600 \mathrm{pC} / \mathrm{N}\right)$ was reported in ceramic samples near this tricritical point. The authors interpret their results in terms of enhanced polarization rotation near the tricritical point where free energies of the cubic, tetragonal and rhombohedral phases become equal. According to the authors, the energy flattening near the tricritical point is more isotropic than in the case of polymorphic or MPB phase transitions, leading to stronger effects of polarization rotation on properties enhancement. While this is true, there is an additional mechanism of properties enhancement in the vicinity of the trictritical point that these authors did not discuss. Near the tricritical point formed by polar rhombohedral and tetragonal phases and nonpolar cubic phase, the free energy flattens along tetragonal-cubic and rhombohedral-cubic paths in addition to rotational tetragonal-rhombohedral path. ${ }^{22,25,63}$ The two former effects lead to enhanced propensity for polarization extension, not rotation. Easy polarization extension can lead to very large enhancement of the piezoelectric properties, in particular near the Curie point. Examples include $\mathrm{KH}_{2} \mathrm{PO}_{4}^{25}$ and $\mathrm{BaTiO}_{3}^{63}$ An example of the temperature-independent polarization extension mechanism can be found in solid solutions forming an MPB between a nonpolar and a polar phase, as reported for lead-free $\mathrm{Al}_{1-x} \mathrm{Sc}_{x} \mathrm{~N}^{64}$ and $\mathrm{Sc}_{1-x} \mathrm{GaN}^{65}$ In these materials, the longitudinal piezoelectric coefficient increases in the MPB region by $400-500 \%$ due to polarization extension mechanisms. In all these cases, the material is softened and free energy flattened along actual or incipient polar directions.

Thus near its tricritical point, $(1-x) \mathrm{Ba}\left(\mathrm{Ti}_{0.8} \mathrm{Zr}_{0.2}\right) \mathrm{O}_{3-}$ $x\left(\mathrm{Ba}_{0.7} \mathrm{Ca}_{0.3}\right) \mathrm{TiO}_{3}$ possesses not only a high susceptibility to polarization rotation (tetragonal rhombohedral transition) but 
also to polarization extension (tetragonal cubic and rhombohedral cubic transitions). The disadvantages of this system are numerous from the practical standpoint: the ferroelectricparaelectric phase transition temperature is less than $<100^{\circ} \mathrm{C}$ across the whole phase diagram; the boundary between the two ferroelectric phases is strongly curved, that is, it possesses a strong polymorphic character; the composition with the highest $d_{33}$ exhibits the Curie point close to the ambient temperature. This material is, however, very interesting from the fundamental point of view as it may be a convenient model system to study factors that contribute to the enhancement of the piezoelectric properties, including: (i) competing effects of the composition and temperature on the flattening and anisotropy of the free energy; (ii) polarization rotation vs. polarization extension effects; and (iii) contributions from the domain walls motion. Practically, the material may be of interest under isothermal conditions.

As mentioned earlier, domain wall contributions have not been investigated in any detail in KNN and BNT systems. This raises the question on whether part of the reason for the comparatively low piezoelectric properties in lead-free materials are due to low domain wall contributions. Our first estimate of domain wall contributions for BNT-BT is given below. We note that this solid solution exhibits a strong relaxor character so that it may be more appropriate to speak of polar nanoregions or polar clusters rather than ferroelectric domains. For simplicity, we shall use here both terms and common term "extrinsic contribution" for effect on the properties.

One way to separate domain wall and lattice contributions to the properties is to study the material's response over a wide frequency or temperature range. ${ }^{66-68}$ Domain wall displacement may lead to a nontrivial dependence of the properties on the driving field frequency. In many ferroelectric materials, a steep dispersion in permittivity and a peak in loss is observed in the frequency region from several hundred $\mathrm{MHz}$ to few $\mathrm{GHz}$. In ferroelectric ceramics, this dispersion in the so-called "microwave" range may be related to the piezoelectric resonance of grains, ${ }^{69}$ acoustic emission of domain walls arranged in a lamellar structure, ${ }^{68,70}$ or to vibration of domain walls. ${ }^{71}$ In addition, a slow, nearly linear decrease of the permittivity with increasing logarithm of frequency is observed in some soft ferroelectric and relaxor materials over a broad frequency range ( $\mathrm{mHz}$ to $\mathrm{GHz}$ ). This frequency dispersion is sometimes referred to as "logarithmic" dispersion. The origin of this weaker dispersion is not clear; in ferroelectrics it is probably related to the motion of domain walls in a random potential profile and a broad distribution of relaxation times. ${ }^{72-74}$ Examples of frequency dispersion of the permittivity for soft, $\left(\mathrm{Pb}\left(\mathrm{Zr}_{0.58} \mathrm{Ti}_{0.42}\right)_{0.99} \mathrm{Nb}_{0.01} \mathrm{O}_{3}\right)$, and hard, $\left(\mathrm{Pb}\left(\mathrm{Zr}_{0.42} \mathrm{Ti}_{0.58}\right)_{0.99} \mathrm{Fe}_{0.01} \mathrm{O}_{3}\right)$, PZT ceramics are shown in Fig. 1. Experimental details are given in Ref. 67.

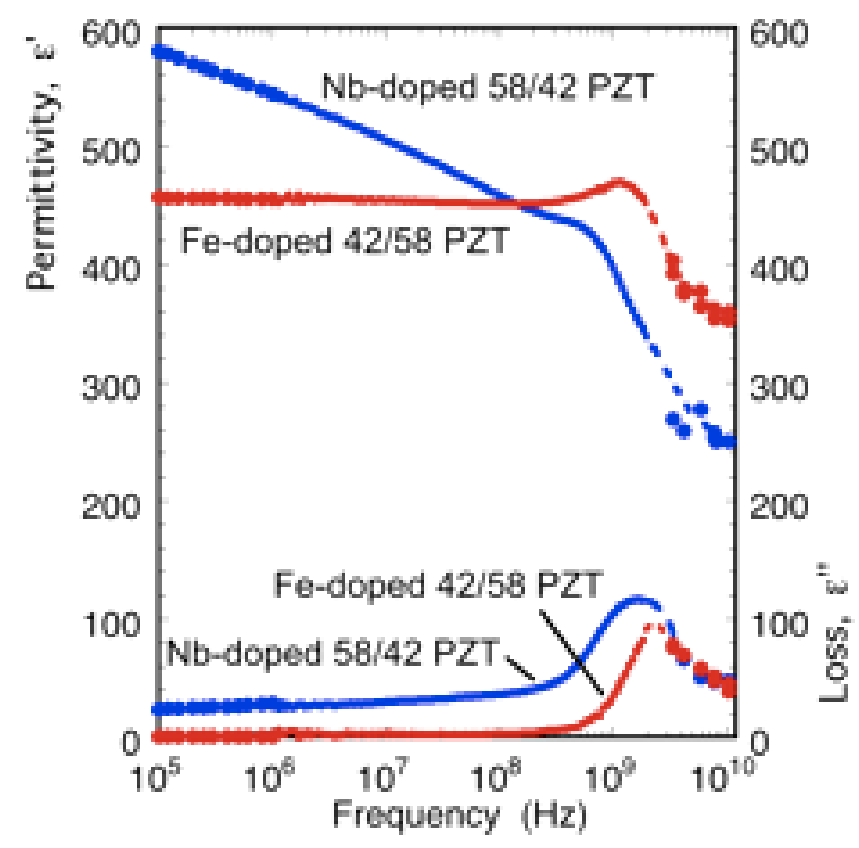

Fig. 1. Permittivity and loss for a soft and a hard PZT ceramics (see text) as a function of frequency. Dashed lines are guides for the eye. For more data, see Ref. 67.

Domain wall contributions may become "frozen" at low temperatures or high frequencies. ${ }^{66,75}$ Therefore, by examining properties over a sufficiently broad temperature or frequency range, it is possible to make estimates of upper and lower limits of domain wall and lattice contributions, as illustrated below. It is common to assume simple superposition of different contributions to the permittivity. In addition, the lattice permittivity is assumed to be independent of the frequency in the frequency range examined here.

We first give a rough estimate of domain wall contributions to the dielectric response of the two PZT ceramics shown in Fig. 1. The peak in loss just above $1 \mathrm{GHz}$ and associated steep permittivity dispersion are dominated by the domain wall process. ${ }^{67}$ If the value of permittivity at $100 \mathrm{kHz}$ is taken as a reference, the permittivity in the soft rhombohedral PZT decreases by more than $34 \%$ in the region of the loss peak. In hard tetragonal PZT ceramics, this decrease is about $23 \%$. The permittivity does not change in hard PZT from $1 \mathrm{GHz}$ to $100 \mathrm{kHz}$, (the small step at $1 \mathrm{MHz}$ is a measurement artifact). In the soft material, there is an additional domain wall contribution which is responsible for the slow "logarithmic" increase of the permittivity with decreasing frequency. This contribution accounts for at least $24 \%$ of the permittivity at $100 \mathrm{kHz}$. Together, the two domain wall contributions account for at least $58 \%$ of the dielectric response of the soft PZT at $100 \mathrm{kHz}$. In the hard PZT, domain wall motion accounts for about $23 \%$ of the permittivity value at $100 \mathrm{kHz}$. 


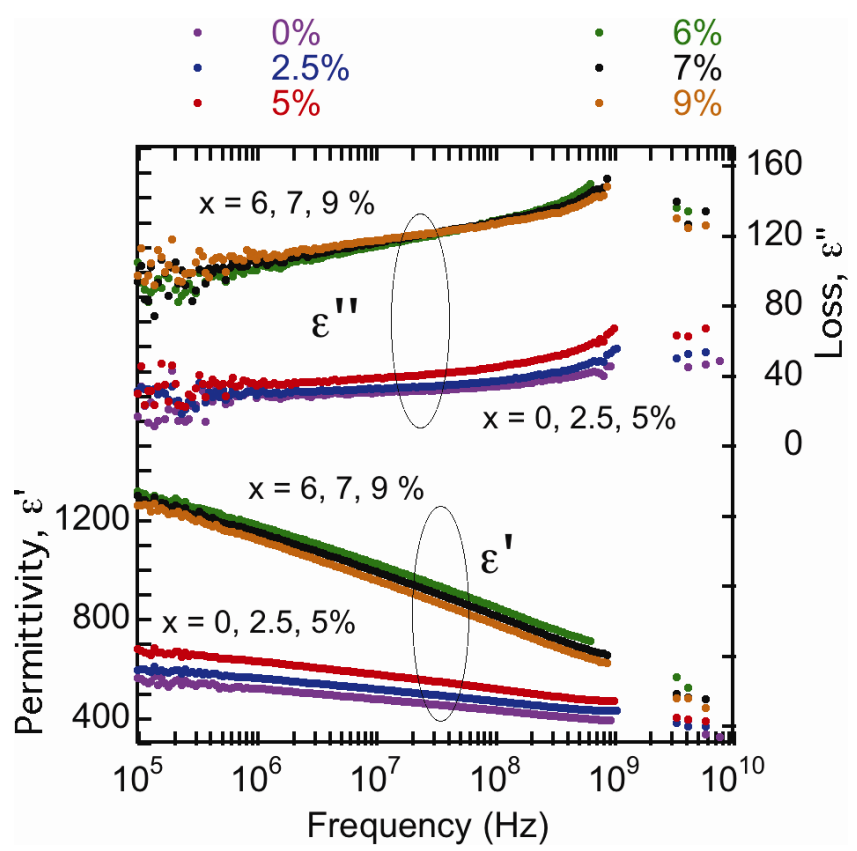

Fig. 2. Dielectric permittivity $\varepsilon^{\prime}$ and loss $\varepsilon^{\prime \prime}$ for $(1-x)$ BNT- $x$ BT for three rhombohedral $(x=0,2.5$ and $5 \%)$ and three tetragonal $(x=6,7$ and $9 \%)$ compositions. The measurements were made on unpoled samples.

We next analyze dispersion in BNT-BT. Figure 2 shows the dielectric permittivity of six compositions of $(1-x)$ BNT$x \mathrm{BT}$ measured at room temperature in the frequency range from $100 \mathrm{kHz}$ to about $6 \mathrm{GHz}$. Measurements were made on three compositions on the rhombohedral $(x=0,2.5$ and 5\%) and three on the tetragonal ( $x=6,7$ and 9\%) side of the MPB. Samples were not poled. Details on measurement procedure and sample preparation can be found in Ref. 54. At high frequencies, permittivities of all samples approximately converge towards 400. The slow "logarithmic" decrease in the permittivity accompanied by a slight increase in loss is clearly visible from $100 \mathrm{kHz}$ to $900 \mathrm{MHz}$ for all compositions. Due to the limitations of the instrument, the data between approximately $900 \mathrm{MHz}$ and $3 \mathrm{GHz}$ could not be taken. Careful inspection of the loss behavior indicates that a peak might be expected in this frequency range for all examined BNT-BT compositions. However, even if this was the case, BNT-BT ceramics would exhibit much weaker "microwave" dispersion compared to PZT. A possible reason for this is that the "microwave" dispersion may require arrangement of domain walls ${ }^{70}$ into regular lamellas. This may not be the case in unpoled BNTBT which exhibits pseudocubic structure in compositions close to MPB ${ }^{55,56}$ and probably not well-developed domain structure. Alternatively, the "microwave" dispersion may be related to vibration of polar cluster boundaries, as reported for $(\mathrm{Pb}, \mathrm{La})(\mathrm{Zr}, \mathrm{Ti}) \mathrm{O}_{3}$ by Rychetsky et al. ${ }^{74}$

On the other hand, the slow "logarithmic" dispersion is stronger in BNT-BT than in soft PZT. One reason for this may be a high disorder of BNT-BT and contribution to the dielectric dispersion of polarization reversal near polar cluster boundaries. ${ }^{74}$ Another is that the temperature of the transition into the nonpolar phase is closer to room temperature in BNTBT $\left(100-180^{\circ} \mathrm{C}\right)$ than in PZT $\left(>350^{\circ} \mathrm{C}\right)$ and the mobility of domain walls/polar cluster boundaries may be higher in BNTBT. Again, taking the value of the permittivity at $100 \mathrm{kHz}$ as the reference, one can infer from Fig. 2 that the total extrinsic contributions account for about $56-63 \%$ of the permittivity in the tetragonal and about $35-41 \%$ in the rhombohedral BNTBT. While these estimates are very rough, they do represent a first indication that contributions to the permittivity from the domain wall motion in BNT-BT are qualitatively different but quantitatively similar to those in soft PZT. It should be noted that the soft PZT used here contains only $\mathrm{Nb}$ as a softener and that commercial soft PZT usually contains other modifiers and possesses higher properties.

We next compare in Fig. 3 the permittivities of the same BNT-BT samples as a function of composition for two frequencies: $1 \mathrm{kHz}$ and $4.2 \mathrm{GHz}$. The permittivity measured at $1 \mathrm{kHz}$ includes lattice contribution and mechanisms responsible for the "logarithmic" frequency dependence and those responsible for the dispersion at "microwave" frequencies. The permittivity measured at $4.2 \mathrm{GHz}$ includes the lattice permittivity and any extrinsic contributions that are not "frozen" at this frequency. The latter therefore represents the upper limit for the lattice permittivity. The difference between the permittivities measured at $1 \mathrm{kHz}$ and $4.2 \mathrm{GHz}, \Delta \varepsilon$, indicates a lower limit for the extrinsic contributions at $1 \mathrm{kHz}$, and is also shown in Fig. 3. Looking at the data from the low $x$ values, the position of the transition from the rhombohedral to the tetragonal phase is clearly marked by the sharp increase in both the permittivity and in extrinsic contributions. In PZT, the MPB transition is characterized by a sharp peak in both dielectric permittivity

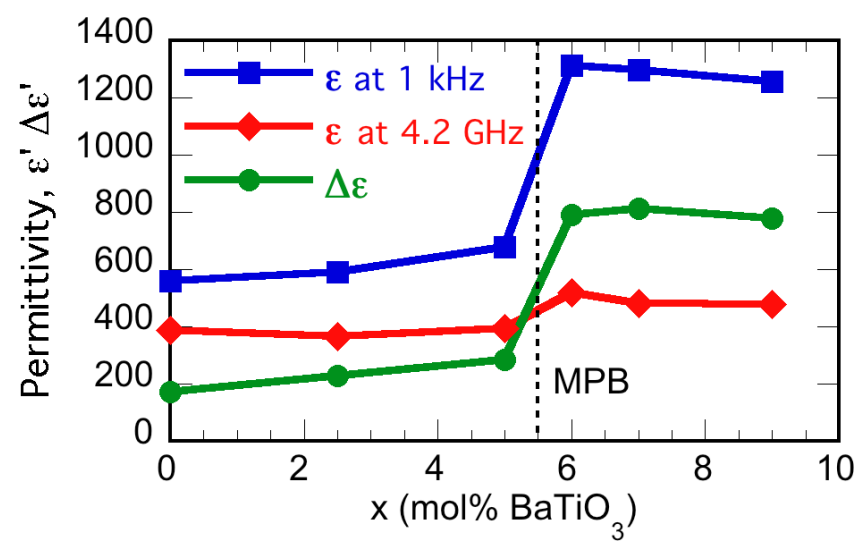

Fig. 3. Dielectric permittivity $\varepsilon$ for $(1-x)\left(\mathrm{Na}_{1 / 2} \mathrm{Bi}_{1 / 2}\right) \mathrm{TiO}_{3}-x \mathrm{BaTiO}_{3}$ ceramics as a function of $x$ at $1 \mathrm{kHz}$ and $4.2 \mathrm{GHz} . \Delta \varepsilon=\varepsilon(1 \mathrm{kHz})-\varepsilon(4.2 \mathrm{GHz})$ represents the lower limit of the domain wall contributions at $1 \mathrm{kHz}$ while $\varepsilon(4.2 \mathrm{GHz})$ represents the upper limit for the lattice permittivity. The data are compiled using measurements shown in Fig. 2. The solid lines are guides for the eye. 
and piezoelectric coupling coefficients. ${ }^{2}$ The peak (not a step) in the permittivity of PZT is intimately connected to a free energy anisotropy at the MPB, as shown by phenomenological calculations. ${ }^{23}$ The absence of a strong peak in BNT-BT may be an indication that the nature of MPBs in PZT and BNT-BT is different. The same type of the step in the dielectric permittivity in BNT-BT was reported by Ranjan et al. ${ }^{56}$ so that experimental artifacts as the reason for the peak absence can probably be excluded. Chen et al. ${ }^{59}$ report a peak in the piezoelectric and electromechanical coupling coefficients in BNT-BT at the MPB, but the peak is weaker than what is normally reported for PZT.

It follows from this simple analysis that the domain wall contributions in BNT-BT are significantly higher on the tetragonal than on the rhombohedral side of the MPB. This is opposite to what was deduced in a study of the nonlinear direct piezoelectric effect in PZT. ${ }^{30}$ That study showed that domain wall contributions in PZT ceramics are higher on the rhombohedral side of the MPB. There are several reasons for this qualitative difference, including the following: (i) it could indicate fundamental differences in the properties of MPB in BNTBT and PZT; (ii) it could be related to the different ways in which non- $180^{\circ}$ (active in both dielectric and piezoelectric response) and $180^{\circ}$ domain walls (active mostly in the dielectric response) contribute to the properties; (iii) the difference could be related to the driving field strength used in the two experiments: weak-field driving and linear response in the case of the dielectric measurements shown in Fig. 3 and subcoercive pressure driving and nonlinear response in the case of the piezoelectric measurements in Ref. 30. Unfortunately, there are no reports on domain wall contributions in PZT and leadfree materials made under comparable conditions. Our own data on soft rhombohedral and hard tetragonal compositions shown in Fig. 1 are in agreement with the study of the piezoelectric effect: the Nb-doped rhombohedral samples exhibit higher domain wall contribution than hard tetragonal material. However, it is probable that the data in Fig. 1 are dominated by the effect of the dopants rather than the structure.

Our results thus point toward qualitative differences in the extrinsic contributions to the electromechanical properties in BNT-BT and PZT ceramics. Further study is required to find out whether these differences are due to the $\mathrm{Pb}$ cation.

Recent studies have shown that both BNT and KNNbased systems do exhibit some advantages over lead-based materials for specific applications. For example, the thickness coupling coefficient $\mathrm{k}_{\mathrm{t}}$ of single crystal $\mathrm{KNbO}_{3}$ and Limodified (K,Na) $\mathrm{NbO}_{3}$ is as large as $70 \% .^{76,77}$ Together with a low permittivity and density, this crystal and its derivatives, make an excellent choice for high frequency single element transducers. ${ }^{78}$ Unfortunately, other piezoelectric properties of $\mathrm{KNbO}_{3}$ are not high enough to make it a viable alternative to PZT for actuators and sensors.
The highest piezoelectric properties in lead free systems at ambient conditions were reported in textured ceramics of $\mathrm{BaTiO}_{3}$ with special domain structure ${ }^{42}$ and in chemically modified and textured KNN. ${ }^{6}$ Textured ceramics are impractical to prepare though being scientifically very interesting and have raised many questions on mechanisms of enhancement of piezoelectric properties in ferroelectrics. The presence (not necessarily motion) of domain walls in domain engineered single crystals and textured and domain engineered ceramics greatly enhances the piezoelectric response of $\mathrm{BaTiO}_{3}{ }^{42}$ These entirely unexpected results obtained in $\mathrm{BaTiO}_{3}$ should encourage further investigation of this effect in other lead-free materials.

\section{Further Development of Lead-Free Piezoelectrics}

Despite considerable worldwide efforts in the last 5-10 years, there are still no viable lead-free alternatives to PZT. Based on the above discussion, one can identify several directions for the future development.

One is to continue search for new lead-free piezoelectric materials empirically, using phenomenological models (e.g. structure-property relations) and first principle calculations. This is a long-term activity that needs to be pursued but its outcome cannot be predicted. The second direction is to improve existing KNN and BNT based materials by chemical modifications and by controlling structural instabilities and extrinsic (e.g. domain wall) contributions. There is a reason to believe that presently reported values of piezoelectric properties in these systems are not optimized and that improvements are possible if compositions are further modified. To do such modifications efficiently, a better structural understanding of these materials is needed. Attempts in this direction are underway and can be especially beneficial for engineering new systems with an MPB..$^{46,50,52,79}$ It is worth mentioning that non-modified PZT compositions also do not necessarily exhibit large piezoelectric properties, even in the MPB region. This is well-illustrated by the first studies of the piezoelectric properties of PZT ceramics in 1950s which report $d_{33}$ piezoelectric coefficient of about $160 \mathrm{pC} / \mathrm{N}$ at MPB. ${ }^{80}$ This value is similar to what is reported currently in most $\mathrm{KNN}$ materials and not much more than in BNT compositions. The present large values of piezoelectric coefficients of $200-600 \mathrm{pC} / \mathrm{N}$ in PZT are achieved by better processing methods, extensive chemical modifications of pure materials and by targeted doping. Thus, KNN and BNT families may still exhibit better properties than those reported at present. ${ }^{14,49,81}$

The domain wall contributions in PZT may account for as much as $70 \%$ of the total piezoelectric effect. These contributions may be effectively increased or decreased by 
donor and acceptor doping. With few exceptions, there is very little data on hardening (acceptor doping) and softening (donor doping) effects or on the estimate of the domain wall contributions in $\mathrm{KNN}^{12}$ and BNT based materials. Domain wall contributions are sensitive to many parameters including concentration and nature of point defects, dopants, and microstructure. In KNN and BNT systems, the stoichiometry is difficult to control ${ }^{82}$ and significant concentration of A-site and oxygen vacancies cannot be excluded. ${ }^{83}$ It is thus not unreasonable to expect that a better control of stoichiometry and microstructure may improve the piezoelectric properties in these materials. It is an encouraging sign that large extrinsic contributions are seen in undoped BNT-BT (Figs. 2 and 3).

\section{Non-Piezoelectric Solution to Electromechanical Coupling}

Finally, for some applications, it is possible to envision a non-piezoelectric solution for the electromechanical coupling. One such possibility is the well-known electrostrictive effect, which was used since the 1980s. ${ }^{1}$ Interestingly, the best electrostrictive materials today are also lead-based (e.g. $\mathrm{Pb}\left(\mathrm{Mg}_{1 / 3} \mathrm{Nb}_{2 / 3}\right) \mathrm{O}_{3}-\mathrm{PMN}$ ), so that electrostrictive solution also requires a radical material change. Since all materials are electrostrictive and only some are piezoelectric, the probability of finding an efficient lead-free electrostrictor might be considerable. Although there is no extensive research done, there is sufficient evidence that some of the considered lead-free systems may exhibit significant electrostrictive strains. ${ }^{61,84}$ Large electromechanical bipolar and unipolar strains $(0.4 \%)$ have been reported in modified BNTBT systems. ${ }^{61,84}$ These strains appear in nonpolar phases, and it is interesting to speculate about their origin. ${ }^{85}$ At least one of the nonpolar phases in BNT-BT phase diagram has been classified as antiferroelectric, but this does not seem to be justified. This phase exhibits some residual piezoelectric effect suggesting a composite structure, with nonpolar matrix and small, probably nanosize dense polar regions which can be reversed by the electric field. These regions are both elastically and electrically active and are probably origin of the restoring force responsible for the pinching of the polarization hysteresis loops and zero residual strain upon field removal. Macroscopically, the electromechanical response resembles field induced antiferroelectric-ferroelectric switching or backswitching reported in Mn-doped BT, ${ }^{86}$ but the microscopic origin is different here and probably takes place on a different scale. The motion of these hypothetical nanoregions represents an extrinsic contribution to the lattice electrostriction, just as displacement of domain walls contributes to the intrinsic lattice piezoelectricity of the ferroelectric phase. A significant strain-field hysteresis which accompanies the strain in modified BNT based materials is disadvantageous for applications. The large unipolar strain could be of interest for "on-off" actuators.

In recent years, another concept in electromechanical coupling has been practically demonstrated. Flexoelectric effect links the strain gradient with polarization or electric field gradient with stress. The effect is not limited to noncentrosymmetric materials, and, surprisingly, it has been demonstrated that the largest effect is observed in lead-free materials. ${ }^{87,88}$ Flexoelectricity is directly proportional to the dielectric permittivity so a combination of materials with a high permittivity and large strain gradient is needed. This opens up a whole range of possibilities to design composite structures with a very large flexoelectric effect: one phase should exhibit a large permittivity and the strain gradient can be then induced by controlling the boundary conditions around this phase. Recently, it has been demonstrated that an electromechanical response larger than in PZT (equivalent $d_{33}$ well over $2000 \mathrm{pC} / \mathrm{N}$ ) can be obtained in composite structures based on $\mathrm{Sr}_{1-x} \mathrm{Ba}_{x} \mathrm{TiO}_{3}$ solid solution. The composite is used to amplify the strain gradient while the ceramic offers large permittivity (about 20,000 at room temperature). ${ }^{89}$ The disadvantage of $\mathrm{SrBaTiO}_{3}$ is that it exhibits high permittivity over a very narrow temperature range (around the Curie temperature). The large and weakly temperature dependent permittivity has been recently reported in $0.82\left[0.94 \mathrm{Bi}_{0.5} \mathrm{Na}_{0.5} \mathrm{TiO}_{3}-0.06 \mathrm{BaTiO}_{3}-\right.$ $0.18 \mathrm{~K}_{0.5} \mathrm{Na}_{0.5} \mathrm{NbO}_{3} .{ }^{84}$ This material may thus be an interesting candidate for flexoelectric composite structures.

\section{Acknowledgement}

Authors acknowledge financial support of the Swiss National Science Foundation Contract No. 200020_124498.

\section{References}

1. K. Uchino, Ferroelectric Devices (Marcel Dekker, New York, 2000).

2. B. Jaffe et al., Piezoelectric Ceramics (Academic, New York, 1971).

3. R. Nevin, Enviro. Res. Sec. A 83, 1 (2000).

4. R. Nevin, Enviro. Res. 104(3), 315 (2007).

5. Official Journal of the European Union, 13.2.2003, L37/19 (2003).

6. Y. Saito et al., Nature 432, 84 (2004).

7. Report for European commission revised by Bosch, EPCOS and Siemens, information about Electrical components which contain Lead in a Glass or Ceramic Matrix Compound (2005), ec.europa.eu/environment/waste/submissions/bosch3.pdf.

8. I. Fuyuno, Nature 435, 1026 (2005).

9. J. Roedel et al., J. Am. Ceram. Soc. 92(6), 1153 (2009).

10. S. Zhang and T. R. Shrout, J. Electroceramics 19(1), 111 (2007).

11. E. Li et al., Jpn. J. Appl. Phys. 47, 7702 (2008).

12. N. M. Hagh et al., IEEE Trans. Ultrason. Ferroelectr. Freq. Control 55, 214 (2008).

13. L. Wu et al., J. Eur. Ceram. Soc. 28(15), 2963 (2008). 
14. S. Zhang et al., Appl. Phys. Lett. 92(15), 152904 (2008).

15. H. Tanaka et al., Phys. Rev. B 74, 172105 (2006).

16. R. E. Cohen, Nature 358, 136 (1992).

17. G. Sághi-Szabó et al., Phys. Rev. Lett. (80), 4321 (1998).

18. P. Baettig et al., Chem. Mater. 17(6), 1376 (2005).

19. E. Cross, Nature 432, 24 (2004).

20. B. Noheda et al., Appl. Phys. Lett. 74(14), 2059 (1999).

21. K. Carl and K. H. Härdtl, Phys. Stat. Sol. (a) 8, 87 (1971).

22. M. Davis et al., J. Appl. Phys. 101(5), 054112 (2007).

23. Y. Ishibashi and M. Iwata, Jpn. J. Appl. Phys. 37 (Part 2, No. 8B), L985 (1998).

24. D. Damjanovic, J. Am. Ceram. Soc. 88(10), 2663 (2005).

25. D. Damjanovic, IEEE Transactions Ultrason. Ferroelectr. Freq. Control 56(8), 1574 (2009).

26. Z. G. Wu and R. E. Cohen, Phys. Rev. Lett. 95(3), 037601 (2005).

27. M. Ahart et al., Nature 451(7178), 545 (2008).

28. I. A. Kornev et al., Phys. Rev. Lett. 95(19), (2005).

29. I. Abrikosov, private communication.

30. D. Damjanovic and M. Demartin, J. Phys. Condens. Matter 9, 4943 (1997).

31. R. Gerson, J. Appl. Phys. 31(1), 188 (1960).

32. M. J. Haun et al., Ferroelectrics 99, 63 (1989).

33. K. A. Schonau et al., Phys. Rev. B 75, 184117 (2007).

34. R. Theissmann et al., J. Appl. Phys. 102(2), (2007).

35. L. Jin et al., Appl. Phys. Lett. 95(1), 012905 (2009).

36. S. Zhang et al., J. Appl. Phys. 104(8), 084103 (2008).

37. M. A. Dubois and P. Muralt, Ferroelectrics 224, 671 (1998).

38. G. Catalan and J. F. Scott, Adv. Mater. 21, 1 (2009).

39. H. Ogihara et al., J. Am. Ceram. Soc. 92(1), 110 (2009).

40. J. Zylberberg et al., Chem. Mater. 19(26), 6385 (2007).

41. F. Jona and G. Shirane, Ferroelectric Crystals (Pergamon, New York, 1962).

42. S. Wada et al., Jpn. J. Appl. Phys. 46, 7039 (2007).

43. T. Karaki et al., Jpn. J. Appl. Phys. 46, L97 (2007).

44. E. Hollenstein et al., J. Eur. Ceram. Soc. 27, 4093 (2007).

45. S. Zhang et al., Appl. Phys. Lett. 91(13), 132913 (2007).

46. J. Tellier et al., Soli. State Sci. 10(2), 177 (2008).

47. E. Hollenstein et al., Appl. Phys. Lett. 87(18), 182905 (2005).

48. Y. P. Guo et al., Mater. Lett. 59(2-3), 241 (2005).

49. E. K. Akdogan et al., Appl. Phys. Lett. 92(11), (2008).

50. N. Klein et al., J. Appl. Phys. 102(1), 014112 (2007).

51. R. E. Jaeger and L. Egerton, J. Am. Ceram. Soc. 45(5), 209 (1962).

52. Y. Hiruma et al., J. Appl. Phys. 104, 124106 (2008).

53. V. Dorcet et al., J. Magn. Magn. Mater. 321, 1758 (2009).

54. N. Klein, $\mathrm{PhD}$ thesis no. 4528, Swiss Federal Institute of Technology - EPFL (2009).
55. J. E. Daniels et al., Appl. Phys. Lett. 95, 032904 (2009).

56. R. Ranjan and A. Dviwedi, Solid State Commun. 135, 394 (2005).

57. T. Takenaka et al., Jpn. J. Appl. Phys. 30(9B), 2236 (1991).

58. D. A. Berlincourt et al., Piezoelectric and piezomagnetic materials and their function in transducers in Physical AcousticsPrinciples and Methods, ed. W.P. Mason Vol. I — Part A (Academic, New York, 1964).

59. M. Chen et al., J. Euro. Ceram. Soc. 2, 843 (2008).

60. Y.-M. Chiang et al., Appl. Phys. Lett. 73(25), 3683 (1998).

61. S. A. Sheets et al., J. Appl. Phys. 90(10), 5287 (2001).

62. W. Liu and X. Ren, Phys. Rev. Lett. 103, 257602 (2009).

63. D. Damjanovic et al., Enhancement of piezoelectric properties in perovskite crystals by thermally, compositionally, electric field and stress induced instabilities in Handbook of Advanced Dielectric Piezoelectric and Ferroelectric Materials: Synthesis, Characterisation and Applications, ed. Z.-G. Ye (Woodhead, Cambridge, 2008), p. 304.

64. M. Akiyama et al., Adv. Mater. 21, 593 (2009).

65. A. Alsaada and A. Ahmad, Eur. Phys. J. B 54, 151 (2006).

66. X. L. Zhang et al., J. Mater. Sci. 18, 968 (1983).

67. V. Porokhonskyy et al., Appl. Phys. Lett. 94, 212906 (2009).

68. N. A. Pertsev and G. Arlt, J. Appl. Phys. 74(6), 4105 (1993).

69. Y. Xi et al., J. Am. Ceram. Soc 66(9), 637 (1983).

70. G. Arlt, IEEE Trans. Ultrason. Ferroelectr. Freq. Control 45(1), 4 (1998).

71. C. Kittel, Phys. Rev. 83, 458 (1951).

72. D. Damjanovic, Phys. Rev. B 55(2), R649 (1997).

73. W. Kleemann et al., Phys. Rev. B 65, 220101 (2002).

74. I. Rychetsky et al., J. Phys. Condens. Matter. 15, 6017 (2003).

75. U. Robels and G. Arlt, J. Appl. Phys. 73(7), 3454 (1993).

76. K. Nakamura et al., J. Appl. Phys. 91(11), 9272 (2002).

77. M. Davis et al., Appl. Phys. Lett. 90(6), 062904 (2007).

78. N. M. Kari et al., in 2000 Ultrason. Symp. (Puertorico, 2000), p. 1065 .

79. H. J. Trodahl et al., Appl. Phys. Lett. 93(26), 262901 (2008).

80. B. Jaffe et al., J. Appl. Phys. 25(6), 809 (1954).

81. M. Matsubara et al., Jpn J. Appl. Phys., Part 1 44(1A), 258 (2005).

82. Y. Wang et al., J. Am. Ceram. Soc. 90(11), 3485 (2007).

83. J. G. Fisher et al., J. Alloys Comp. 479(1-2), 467 (2009).

84. S. T. Zhang et al., Advan. Mater. 21, 4716 (2009).

85. W. Jo et al., J. Appl. Phys. 105(9), 094102 (2009).

86. X. B. Ren, Nat. Mater. 3(2), 91 (2004).

87. J. Y. Fu et al., Appl. Phys. Lett. 91(18), 182910 (2007).

88. J. Y. Fu and L. E. Cross, Ferroelectrics 354, 238 (2007).

89. B. Chu et al., J. Appl. Phys. 106(10), 104109 (2009). 